\title{
Non-Markovian renormalization of kinetic coefficients for drift wave turbulence
}

\author{
A.Zagorodny ${ }^{1}$, J.Weiland ${ }^{2}$ \\ 1 Bogolyubov Institute for Theoretical Physics, 03143 Kiev 143, Ukraine \\ 2 Institute for Electromagnetics, Chalmers University of Technology, \\ S-41296 Göteborg, Sweden
}

Received August 29, 2001

\begin{abstract}
Statistical theory of non-Markovian effects in turbulent transport is applied to the description of plasma turbulence in a magnetoactive plasma in the drift-kinetic approximation. Renormalized transition probability is calculated with regard to non-Markovian effects and equations for the relevant kinetic coefficients are derived. It is shown that memory effects can be important for the description of transport under saturated turbulence.
\end{abstract}

Key words: plasma turbulence, drift-kinetic approximation, non-Markovian effects

PACS: 05.40. $+j$, 52.35.Ra, 25.65.Ff

\section{Introduction}

In $[1,2]$ we proposed statistical theory of turbulent transport giving the nonMarkovian version of the Dupree-Weinstock renormalization [3,4]. In the present paper the transition probability approach is used to introduce non-Markovian generalization of drift kinetic equations with regard to turbulent collisions. Derived equations include collision terms describing the transverse diffusion in the real space and longitudinal diffusion in the velocity space. The main feature of the non-Markovian description is the time-nonlocality of the collision terms, which leads to a frequency dependence of transport kinetic coefficients the real part of which describes the modification of the growthrate while the imaginary part is responsible for coherent wave interaction, in particular, for nonlinear frequency shift.

The proposed approach makes it possible to calculate the renormalized transition probability taking into account the influence of turbulent fields on the particle trajectories. It is shown that non-Markovian effects could be important for turbulence saturation. In the Markovian limit the Dupree-Tetreault renormalization [5] is reproduced. 


\section{Drift kinetic equation with turbulent collision term}

We start from the equation for the microscopic phase density of guiding centers

$$
N(X, t)=\sum_{i=1}^{N} \delta\left(X-X_{i}(t)\right), \quad X=\left(\mathbf{r}, v_{\|}\right)
$$

which in the drift approximation is given by

$$
\left\{\frac{\partial}{\partial t}+\left(\mathbf{v}_{g}+\mathbf{v}_{E}\right) \frac{\partial}{\partial \mathbf{r}_{\perp}}+v_{\|} \frac{\partial}{\partial r_{\|}}+\frac{e}{m} E_{\|} \frac{\partial}{\partial v_{\|}}\right\} N(X, t)=0 .
$$

Here $\mathbf{v}_{E}=\frac{e c}{B_{0}}\left[\mathbf{E}_{\perp} \cdot \mathbf{e}_{\|}\right], \mathbf{E}=-\operatorname{grad} \Phi$ is the microscopic electric field, $\mathbf{v}_{g}$ is the gravitation drift velocity, the other notation is conventional.

The kinetic equation for the distribution function $f(X, t) \equiv\langle N(X, t)\rangle$ can be obtained by statistical averaging of equation (2)

$$
\hat{L}^{0} f(X, t) \equiv\left\{\frac{\partial}{\partial t}+\left(\mathbf{v}_{g}+\left\langle\mathbf{v}_{E}\right\rangle\right) \frac{\partial}{\partial \mathbf{r}_{\perp}}+v_{\|} \frac{\partial}{\partial r_{\|}}+\frac{e}{m}\left\langle E_{\|}\right\rangle \frac{\partial}{\partial v_{\|}}\right\} f(X, t)=I,
$$

where

$$
\begin{aligned}
I & =I_{\perp}+I_{\|}, \\
I_{\|} & =-\frac{e}{m} \frac{\partial}{\partial v_{\|}}\left\langle\delta E_{\|} \delta N\right\rangle, \quad I_{\perp}=-\frac{\partial}{\partial \mathbf{r}_{\perp}}\left\langle\delta \mathbf{v}_{E} \delta N\right\rangle .
\end{aligned}
$$

The equation for fluctuations reduces then to

$$
\left\{\hat{L}^{0}+\frac{e}{m} \delta E_{\|}(\mathbf{r}, t) \frac{\partial}{\partial v_{\|}}\right\} \delta N(X, t)=-\delta \mathbf{v}_{E}(\mathbf{r}, t) \frac{\partial f(X, t)}{\partial \mathbf{r}_{\perp}}-\frac{e}{m} \delta E_{\|}(\mathbf{r}, t) \frac{\partial f(X, t)}{\partial v_{\|}} .
$$

The formal solution of equation (5) is given by

$$
\begin{aligned}
& \delta N(X, T)=\delta N^{(0)}(X, t) \\
& \quad-\int_{0}^{t} \mathrm{~d} t^{\prime} \int \mathrm{d} X^{\prime} W_{m}\left(X, X^{\prime} ; t, t^{\prime}\right)\left[\delta \mathbf{v}_{E}\left(\mathbf{r}^{\prime}, t^{\prime}\right) \frac{\partial f\left(X^{\prime}, t^{\prime}\right)}{\partial \mathbf{r}_{\perp}^{\prime}}+\frac{e}{m} \delta E_{\|}\left(\mathbf{r}^{\prime}, t^{\prime}\right) \frac{\partial f\left(X^{\prime}, t^{\prime}\right)}{\partial v_{\|}^{\prime}}\right],
\end{aligned}
$$

where $\delta N^{(0)}(X, t)$ is the fluctuation of the microscopic phase density with no selfconsistent interaction generated by the general solution of homogeneous equation, i.e.

$$
\left\{\hat{L}^{(0)}+\frac{e}{m} \delta E_{\|}(\mathbf{r}, t) \frac{\partial}{\partial v_{\|}}\right\} \delta N^{0}(X, t)=0
$$

$W_{m}\left(X, X^{\prime} ; t, t^{\prime}\right)$ is the "microscopic" probability of particle transition from $X^{\prime}$ to $X$ during the time interval $t-t^{\prime}$. Obviously the equation for such probability is

$$
\left\{\hat{L}^{(0)}+\frac{e}{m} \delta E_{\|}(\mathbf{r}, t) \frac{\partial}{\partial v_{\|}}\right\} W_{m}\left(X, X^{\prime} ; t, t^{\prime}\right)=0
$$


with the initial condition $W_{m}\left(X, X^{\prime} ; t, t^{\prime}\right)=\delta\left(X-X^{\prime}\right)$. The solution of equation (8) is

$$
W_{m}\left(X, X^{\prime} ; t, t^{\prime}\right)=\delta\left(X-X^{\prime}-\Delta X\left(X^{\prime}, t^{\prime} ; t\right)\right),
$$

where $\Delta X\left(X^{\prime}, t^{\prime} ; t\right)$ is the guiding center displacement in the course of particle motion in the microfield

$$
\begin{aligned}
& \Delta \mathbf{r}_{\|}\left(X^{\prime}, t^{\prime} ; t\right)=v_{\|}\left(t^{\prime}\right)\left(t-t^{\prime}\right)+\frac{e}{m} \int_{t^{\prime}}^{t} \mathrm{~d} s \int_{t^{\prime}}^{s} \mathrm{~d} s^{\prime} E_{\|}\left(\mathbf{r}\left(s^{\prime}\right), s^{\prime}\right), \\
& \Delta \mathbf{r}_{\perp}\left(X^{\prime}, t^{\prime} ; t\right)=\mathbf{v}_{g}\left(t-t^{\prime}\right)+\int_{t^{\prime}}^{t} \mathrm{~d} s \mathbf{v}_{E}(\mathbf{r}(s), s), \\
& \Delta v_{\|}\left(X^{\prime}, t^{\prime} ; t\right)=\frac{e}{m} \int_{t^{\prime}}^{t} \mathrm{~d} s E_{\|}(\mathbf{r}(s), s) .
\end{aligned}
$$

We now assume that the distribution function slowly changes within the spatial and velocity fluctuation scales. Then, combining equations (4), (6) and (8) it is possible to introduce the following kinetic equation with the non-Markovian collision term

$$
\begin{gathered}
\hat{L}^{(0)} f(X, t)=\int_{0}^{t} \mathrm{~d} t^{\prime}\left\{\frac{\partial}{\partial v_{\|}}\left[v_{\|}\left(\beta\left(t, t^{\prime}, v_{\|}\right)+2 \mathbf{U}^{f}\left(t, t^{\prime}, v_{\|}\right) \frac{\partial}{\partial \mathbf{r}_{\perp}}\right)+\frac{\partial}{\partial v_{\|}} d\left(t, t^{\prime}, v_{\|}\right)\right]\right. \\
\left.-\left(\mathbf{U}^{P}\left(t, t^{\prime}, v_{\|}\right)+\mathbf{U}^{f}\left(t, t^{\prime}, v_{\|}\right)\right) \frac{\partial}{\partial \mathbf{r}_{\perp}}+D_{i j}\left(t, t^{\prime}, v_{\|}\right) \frac{\partial^{2}}{\partial r_{\perp i} \partial r_{\perp j}}\right\} f\left(X, t^{\prime}\right),
\end{gathered}
$$

where

$$
\begin{aligned}
& \beta\left(t, t^{\prime}, v_{\|}\right)=\beta_{1}\left(t, t^{\prime}, v_{\|}\right)+\beta_{2}\left(t, t^{\prime}, v_{\|}\right), \\
& \beta_{1}\left(t, t^{\prime}, v_{\|}\right)=-\mathrm{i} \frac{4 \pi e^{2} n}{m} \int \frac{\mathrm{d} \mathbf{k}}{(2 \pi)^{3}} \int \frac{\mathrm{d} \omega}{2 \pi} \frac{k_{i}\left\langle W_{m \mathbf{k} \omega}\left(v_{\|}\right)\right\rangle}{k^{2} \varepsilon(\mathbf{k}, \omega)} \delta\left(t-t^{\prime}\right)+\text { c.c. }, \\
& \beta_{2}\left(t, t^{\prime}, v_{\|}\right)=-\frac{1}{v_{\|}} \frac{\partial}{\partial v_{\|}}\left(\frac{e}{m}\right)^{2} \int \frac{\mathrm{d} \mathbf{k}}{(2 \pi)^{3}} k_{\|}^{2}\left\langle\delta \Phi(t) \delta \Phi\left(t^{\prime}\right)\right\rangle_{\mathbf{k}}\left\langle W_{m \mathbf{k}}\left(v_{\|}, t^{\prime} ; t\right)\right\rangle, \\
& U_{i}^{P}\left(t, t^{\prime} v_{\|}\right)=-\mathrm{i} \frac{4 \pi e^{2}}{m \Omega} e_{i j z} \int \frac{\mathrm{d} \mathbf{k}}{(2 \pi)^{3}} \int \frac{\mathrm{d} \omega}{2 \pi} \frac{k_{\perp i}\left\langle W_{m \mathbf{k} \omega}\left(v_{\|}\right)\right\rangle}{k^{2} \varepsilon(\mathbf{k}, \omega)} \delta\left(t-t^{\prime}\right)+\text { c.c. }, \\
& U_{i}^{f}\left(t, t^{\prime}, v_{\|}\right)=e_{i j z}\left(\frac{e}{m}\right)^{2} \int \frac{\mathrm{d} \mathbf{k}}{(2 \pi)^{3}} \frac{k_{\perp j}}{\Omega v_{\|}}\left\langle\delta \Phi(t) \delta \Phi\left(t^{\prime}\right)\right\rangle_{\mathbf{k}}\left\langle W_{m \mathbf{k}}\left(v_{\|}, t^{\prime} ; t\right)\right\rangle, \\
& d\left(t, t^{\prime} ; v_{\|}\right)=\left(\frac{e}{m}\right)^{2} \int \frac{\mathrm{d} \mathbf{k}}{(2 \pi)^{3}} k_{\|}^{2}\left\langle\delta \Phi(t) \delta \Phi\left(t^{\prime}\right)\right\rangle_{\mathbf{k}}\left\langle W_{m \mathbf{k}}\left(v_{\|}, t^{\prime} ; t\right)\right\rangle, \\
& D_{i j}\left(t, t^{\prime} ; v_{\|}\right)= \\
& =\left(\frac{e}{m}\right)^{2} \int \frac{\mathrm{d} \mathbf{k}}{(2 \pi)^{3}} \frac{k_{\perp}^{2}}{\Omega^{2}}\left(\delta_{i j}^{\perp}-\frac{k_{\perp i} k_{\perp j}}{k_{\perp}^{2}}\right)\left\langle\delta \Phi(t) \delta \Phi\left(t^{\prime}\right)\right\rangle_{\mathbf{k}}\left\langle W_{m \mathbf{k}}\left(v_{\|}, t^{\prime} ; t\right)\right\rangle
\end{aligned}
$$


and

$$
\begin{aligned}
& \left\langle W_{m \mathbf{k}}\left(v_{\|}, t, t^{\prime}\right)\right\rangle=\int \mathrm{d} \Delta X \mathrm{e}^{-\mathrm{i} \mathbf{k} \Delta \mathbf{r}}\left\langle W\left(X+\Delta X, X ; t, t^{\prime}\right)\right\rangle, \\
& \left\langle W_{m \mathbf{k} \omega}\left(v_{\|}\right)\right\rangle=\int_{0}^{\infty} \mathrm{d} \tau \mathrm{e}^{\mathrm{i} \omega \tau}\left\langle W_{m k}\left(v_{\|} ; t+\tau, t\right)\right\rangle, \\
& \varepsilon(k, \omega)=1-\frac{4 \pi e^{2}}{m} \int \mathrm{d} v_{\|}\left\langle W_{m \mathbf{k} \omega}\left(v_{\|}\right)\right\rangle\left\{e_{i j z} \frac{k_{\perp j}}{\Omega} \frac{\partial f(X, t)}{\partial r_{\perp i}}+k_{\|} \frac{\partial f(X, t)}{\partial v_{\|}}\right\} .
\end{aligned}
$$

The kinetic coefficents given by equation (11) describe the influence of different physical mechanisms on averaged (kinetic) particle dynamics. Namely, $\beta_{1}\left(t, t^{\prime} ; v_{\|}\right)$ and $\beta_{2}\left(t, t^{\prime} ; v_{\|}\right)$describe the polarization friction and friction due to the particle scattering by turbulent fields, respectively, $U^{p}\left(t, t^{\prime} ; v_{\|}\right)$and $U^{f}\left(t, t^{\prime} ; v_{\|}\right)$are associated with the polarization drift and zonal flows, $d\left(t, t^{\prime} ; v_{\|}\right)$and $D\left(t, t^{\prime} ; v_{\|}\right)$characterize the velocity diffusion along the external magnetic field and the transverse diffusion in the real space. Thus, we see that in the case under consideration the collision term in equation (10) includes diffusion in the velocity space in longitudinal direction, diffusion in the real space in the transverse directions, drift generated by the polarization forces and drift due to the field fluctuations. All kinetic coefficients are expressed in terms of fluctuation potential correlation functions and averaged transition probability which describes particle transitions taking into account fluctuation (in particular turbulent) field influence on particle trajectories.

\section{Renormalized transition probability and dielectric response function}

Since the equation for "microscopic" transition probability (equation (8)) coincides with the equation for microscopic phase density (equation (2)), it is easy to derive the equation for averaged transition probability given by

$$
\begin{aligned}
& \hat{L}^{0}\left\langle W_{m}\left(X, X^{\prime} ; t, t^{\prime}\right)\right\rangle= \\
& =\int_{t^{\prime}}^{t} \mathrm{~d} t^{\prime \prime}\left\{\frac{\partial}{\partial v_{\|}}\left[v_{\|}\left(\beta\left(t, t^{\prime \prime}, v^{\|}\right)+2 \mathbf{U}^{f}\left(t, t^{\prime \prime}, v_{\|}\right)+\frac{\partial}{\partial \mathbf{r}_{\perp}}\right) \frac{\partial}{\partial v_{\|}} d\left(t, t^{\prime} ; v_{\|}\right)\right]\right. \\
& \left.\quad-\left(\mathbf{U}^{P}\left(t, t^{\prime}, v_{\|}\right)+\mathbf{U}^{f}\left(t, t^{\prime \prime} ; v_{\|}\right)\right) \frac{\partial}{\partial \mathbf{r}_{\perp}}+D_{i j}\left(t, t^{\prime \prime} ; v^{\|}\right) \frac{\partial^{2}}{\partial r_{\perp i} \partial r_{\perp j}}\right\} \\
& \quad \times\left\langle W_{m}\left(X, X^{\prime} ; t^{\prime \prime}, t^{\prime}\right)\right\rangle .
\end{aligned}
$$

This equation, as well as the kinetic equation (10) are non-Markovian. In various particular cases solutions of equation (13) can be found explicitly. The examples of solutions of the equation describing non-Markovian diffusion in the velocity space are given in $[1,2]$. We generalize these results to the case under consideration, i.e. to equation (13). However, in order to illustrate the basic points of the approach, 
we do not reproduce here the general solution but restrict ourselves to the case when the dynamical friction, polarization and fluctuation drift, could be neglected $\left(\beta=0, \mathbf{U}^{P}=\mathbf{U}^{f}=0\right)$. This approximation is valid in the case of wide $k$-spectrum of turbulence and low level of turbulent fluctuations.

In this case equation (13) reduces to

$$
\begin{aligned}
& \hat{L}^{0}\left\langle W_{m}\left(X, X^{\prime} ; t, t^{\prime}\right)\right\rangle= \\
& \quad=\int_{t^{\prime}}^{t} \mathrm{~d} t^{\prime \prime}\left[d\left(t, t^{\prime \prime}, v_{\|}\right) \frac{\partial^{2}}{\partial v_{\|}^{2}}+D_{i j}\left(t, t^{\prime \prime} ; v_{\|}\right) \frac{\partial^{2}}{\partial r_{\perp i} \partial r_{\perp j}}\right]\left\langle W_{m}\left(X, X^{\prime} ; t^{\prime \prime}, t^{\prime}\right)\right\rangle .
\end{aligned}
$$

It follows from the solution of this equation that in the case of stationary system

$$
\begin{aligned}
& \left\langle W_{m \mathbf{k} \omega}\left(v_{\|}\right)\right\rangle= \\
& \quad=\int_{0}^{\infty} \mathrm{d} \tau \exp \left[\mathrm{i}\left(\omega-k_{\|} v_{\|}-\mathbf{k}_{\perp} \mathbf{v}_{g}+\mathrm{i} k_{\perp i} k_{\perp j} D_{i j \omega}\left(v_{\|}\right)\right) \tau+\frac{\mathrm{i} k_{\|}^{2} d_{\omega}\left(v_{\|}\right)}{3} \tau^{3}\right],
\end{aligned}
$$

where

$$
d_{\omega}\left(v_{\|}\right)=\int_{0}^{\infty} \mathrm{d} \tau \mathrm{e}^{\mathrm{i} \omega \tau} d\left(t+\tau, t ; v_{\|}\right), \quad D_{i j \omega}\left(v_{\|}\right)=\int_{0}^{\infty} \mathrm{d} \tau \mathrm{e}^{\mathrm{i} \omega \tau} D_{i j}\left(t+\tau, t ; v_{\|}\right) .
$$

In the Markovian limit these quantities should be replaced by their zero-frequency values. Equation (15) generalizes the renormalizations of the transition probability for guiding centers due to the diffusion in the real and velocity spaces to the case of non-Markovian diffusion.

The renormalized dielectric response function has the form

$$
\begin{aligned}
\varepsilon(k, \omega)= & -\mathrm{i} \frac{4 \pi e^{2}}{m} \int \mathrm{d} v_{\|} \int_{0}^{\infty} \mathrm{d} \tau \\
& \times \exp \left[\mathrm{i}\left(\omega-k_{\|} v_{\|}-\mathbf{k}_{\perp} \mathbf{v}_{g}+\mathrm{i} k_{\perp i} k_{\perp j} D_{i j \omega}\left(v_{\|}\right)\right) \tau+\frac{k_{\|}^{2} d_{\omega}\left(v_{\|}\right)}{3} \tau^{3}\right] \\
& \times\left\{e_{i j z} \frac{k_{\perp j}}{\Omega} \frac{\partial f(X)}{\partial r_{i}}+k_{\|} \frac{\partial f(X)}{\partial v_{\|}}\right\} .
\end{aligned}
$$

Solutions of the equation $\varepsilon(k, \omega)=0$ define the renormalized spectra of eigenexcitations.

\section{Non-Markovian transport and turbulence saturation}

Substituting equation (15) into equations (11) it is possible to formulate equations for renormalized kinetic coefficients. For examples,

$$
D_{i j \omega}\left(v_{\|}\right)=\left(\frac{e}{m}\right)^{2} \int \frac{\mathrm{d} \mathbf{k}}{(2 \pi)^{3}} \int \frac{\mathrm{d} \omega^{\prime}}{2 \pi} \frac{k_{\perp}^{2}}{\Omega^{2}}\left(\delta_{i j}^{\perp}-\frac{k_{\perp i} k_{\perp j}}{k_{\perp}^{2}}\right)\left\langle\delta \Phi^{2}\right\rangle_{\mathbf{k} \omega^{\prime}}
$$




$$
\times \int_{0}^{\infty} \mathrm{d} \tau \exp \left[\mathrm{i}\left(\omega-\omega^{\prime}+k_{\|} v_{\|}+\mathbf{k}_{\perp} \mathbf{v}_{g}+\mathrm{i} k_{\perp i} k_{\perp j} D_{i j \omega-\omega^{\prime}}\left(v_{\|}\right)\right) \tau-\frac{k_{\|}^{2} d_{\omega-\omega^{\prime}}\left(v_{\|}\right)}{3} \tau^{3}\right] .
$$

Within the approximation

$$
\int_{0}^{\infty} \mathrm{d} \tau \mathrm{e}^{-\alpha \tau^{3}+\mathrm{i} \omega \tau} \simeq \frac{\mathrm{i}}{\omega+\mathrm{i} \alpha^{1 / 3}}
$$

equation (17) reduces to

$$
\begin{aligned}
D_{i j \omega}\left(v_{\|}\right)= & \left(\frac{e}{m}\right)^{2} \int \frac{\mathrm{d} \mathbf{k}}{(2 \pi)^{3}} \int \frac{\mathrm{d} \omega^{\prime}}{2 \pi} \frac{k_{\perp}^{2}}{\Omega^{2}}\left(\delta_{i j}^{\perp}-\frac{k_{\perp i} k_{\perp j}}{k_{\perp}^{2}}\right)\left\langle\delta \Phi^{2}\right\rangle_{\mathbf{k} \omega^{\prime}} \\
& \times \frac{\mathrm{i}}{\omega-\omega^{\prime}+k_{\|} v_{\|}+\mathrm{i} k_{\perp i} k_{\perp j} D_{i j \omega-\omega^{\prime}}\left(v_{\|}\right)+\mathrm{i} \sqrt[3]{\frac{k_{\|}^{2} d_{\omega-\omega^{\prime}}\left(v_{\|}\right)}{3}}} .
\end{aligned}
$$

At $d_{\omega}\left(v_{\|}\right)=0, k_{\|} v_{\|}=0$ equation (18) gives the non-Markovian generalization of the result by Dupree-Tetreault [5]

$$
D_{i j \omega}\left(v_{\|}\right)=\left(\frac{e}{m}\right)^{2} \int \frac{\mathrm{d} \mathbf{k}}{(2 \pi)^{3}} \int \frac{\mathrm{d} \omega^{\prime}}{2 \pi} \frac{k_{\perp}^{2}}{\Omega^{2}}\left(\delta_{i j}^{\perp}-\frac{k_{\perp i} k_{\perp j}}{k_{\perp}^{2}}\right) \frac{\mathrm{i}\left\langle\delta \Phi^{2}\right\rangle_{\mathbf{k} \omega^{\prime}}}{\omega-\omega^{\prime}+\mathrm{i} k_{\perp i} k_{\perp j} D_{i j \omega-\omega^{\prime}}} .
$$

In the case of one-mode turbulent spectrum equation (19) gives

$$
k_{\perp i} k_{\perp j} D_{i j \omega}=\frac{1}{2} \sum_{\beta= \pm 1} \frac{k_{\perp}^{4}}{\Omega^{2}} \frac{\Delta k}{k_{\perp}} \frac{\mathrm{i}\left(\frac{e}{m}\right)^{2} \delta \Phi^{2}}{\omega-\beta \omega_{\mathbf{k}}+\mathrm{i} k_{\perp i} k_{\perp j} D_{i j \omega-\beta \omega_{\mathbf{k}}}}
$$

where $\mathbf{k}_{\perp}$ and $\Delta k$ are the wave number related to the maximum growthrate and the turbulent spectrum width, $\omega_{\mathbf{k}}$ is the eigenfrequency, $\delta \Phi^{2}$ is the turbulent spectrum amplitude.

As follows from equation (20) low-frequency value of the diffusion coefficient is given by

$$
k_{\perp i} k_{\perp j} D_{i j}=\frac{\left(\frac{e}{m}\right)^{2} \frac{k_{\perp}^{2}}{\Omega^{2}} \frac{\Delta k}{k_{\perp}} \delta \Phi^{2} \gamma_{\mathbf{k}}}{\omega_{k}^{2}+\gamma_{k}^{2}} \simeq \frac{\gamma_{k}^{3}}{\omega_{k}^{2}+\gamma_{k}^{2}}
$$

where $\gamma_{\mathbf{k}}$ is the instability growthrate. When deriving (21) we assume that in the saturated state renormalized growthrate is equal to zero, i.e. $k_{\perp i} k_{\perp j} D_{\omega_{\mathbf{k}}}=\gamma_{k}$. The estimate (21) is in agreement with the results of mode-coupling simulations and improved mixing length transport theory (see, for instance [6]).

\section{Summary and conclusions}

The microscopic derivation of the drift-kinetic equation with a non-Markovian collision term is done for the case of turbulent plasmas in an external magnetic 
field. Renormalized non-Markovian kinetic coefficients are expressed in terms of the transition probability calculated with regard to the time-nonlocal diffusion in the real and velocity spaces. In the approximation of the time-local diffusion the results of the quasilinear theory, as well as the Dupree-Tetreault renormalization are reproduced. It is shown that memory effects are important for the estimates of the diffusion coefficients for saturated turbulence.

\title{
References
}

1. Zagorodny A., Weiland J. // Condens. Matter Phys., 1998, vol. 1, p. 835.

2. Zagorodny A., Weiland J. // Phys. Plasmas, 1999, vol. 5, p. 2359.

3. Dupree T. H. // Phys. Fluids, 1966, vol. 9, p. 1773.

4. Weinstock J. // Phys. Fluids, 1969, vol. 12, p. 1045.

5. Dupree T. H., Tetreault D. // Phys. Fluids, 1978, vol. 21, p. 425

6. Connor J.W., Pogutse O.P. // Plasma Phys. Control. Fusion, 2001, vol. 43, p. 155.

\section{Немарковське перенормування кінетичних коефіцієнтів для дрейфової турбулентності}

\author{
А.Загородній ${ }^{1}$, Я.Вейланд ${ }^{2}$ \\ 1 Інститут теоретичної фізики ім. М.М. Боголюбова НАН України, \\ 03143 Київ, вул. Метрологічна, 146 \\ 2 Інститут електромагнетизму, \\ Чалмерський технологічний університет, \\ S-41296 Ґетеборґ, Швеція
}

Отримано 29 серпня 2001 р.

Статистична теорія немарковських ефектів у процесах турбулентного перенесення застосована до опису насичення плазмової турбулентності плазми в зовнішньому магнітному полі. Розраховано перенормовану ймовірність переходу з урахуванням немарковських ефектів і сформульовано рівняння для перенормованих кінетичних коефіцієнтів. Показано, що ефекти пам'яті можуть бути суттєвими при описі насичення турбулентності.

Ключові слова: турбулентність плазми, дрейфове кінетичне наближення, немарковські ефекти

PACS: $05.40 .+j, 52.35 . R a, 25.65 . F f$ 
